\title{
Using GoPro to create an educational database of open urological procedures for residents
}

\author{
Landan MacDonald ${ }^{1}$; Jesse Ory ${ }^{2}$; Ricardo A. Rendon ${ }^{2}$; Gregory Bailly²; Thomas Skinner²; \\ Ashley Cox ${ }^{2}$; Gavin Langille ${ }^{2,3}$; David Bell ${ }^{2}$ \\ ${ }^{1}$ School of Medicine, Dalhousie University, St. John, NB, Canada; ${ }^{2}$ Department of Urology, Dalhousie University, \\ Halifax, NS, Canada; ${ }^{3}$ Horizon Health Network, Saint John, NB, Canada
}

Cite as: MacDonald L, Ory J, Rendon RA, et al. Using GoPro to create an educational database of open urological procedures for residents. Can Urol Assoc J 2020 November 17; Epub ahead of print. http://dx.doi.org/10.5489/cuaj.6768

Published online November 17, 2020

$* * *$

\section{Introduction}

The use of surgical video recording is an invaluable educational tool that is not frequently used in residency programs across Canada. Video-assisted laparoscopy and robotics have afforded a medium that makes recordings easy, as evidenced by the numerous websites available to surgeons and trainees. ${ }^{1}$ Although less prevalent than it once was, open surgery still represents a significant proportion of the average Canadian urologists' operative time, ${ }^{2}$ and there are significant challenges to integrating video recording into this medium.

Commercial video capturing devices can cost up to $\$ 10,000$ USD and do not necessarily produce high quality results. ${ }^{3}$ Camera placement, lighting, and surgeon movement have impeded the ability to capture video in open procedures as well. As a cost-saving measure, several groups have examined the use of $\mathrm{GoPro}^{\mathrm{TM}}$ cameras in the operating room to record surgical procedures. ${ }^{3-8}$ Originally marketed as an action camera for sports enthusiasts, the GoPro brand has become popular in the healthcare setting due to its low cost, light weight, large storage capacity, ease of use, and high-quality video recordings in the operating room setting. To our knowledge, we describe the first experience of using GoPro footage to create a database of open urological procedures.

\section{Methods}

Using a GoPro Hero 5 package marketed towards operating room use (Figure 1), we aimed to create a secure database, only accessible by the Department of Urology, Dalhousie University residents/students, with videos of each major operation performed by each surgeon. Two residents and one attending have worn the device. Twelve attendings have agreed to have their surgeries recorded, and six have had surgeries recorded thus far. 
The GoPro Hero 5 is paired with a 128GB microSD card. Cases are recorded in 1080p at $60 \mathrm{fps}$ which allows for approximately 350 minutes of total record time. The device has a battery life of 10 hours due to a battery pack included with the package, which is kept on a hip pack so movement during the case is unimpeded. With these specifications, practically any urological procedure can be recorded without interruption. Prior to each case, a media use consent form (Supplementary Material 1) is obtained from the patient. The GoPro is secured to the resident's or attending's head using the included adjustable head mount. The correct field of view is optimized by using the GoPro phone app.

Following video acquisition, the video files are edited using Adobe Premier Pro or Da Vinci Resolve to exclude any identifiable information. Once edited, videos are uploaded to a Microsoft SharePoint folder on an internal University server. Key steps in each procedure are listed in the description section of the video. An anonymous survey was sent to all residents and staff to collect feedback on the use of the GoPro in the operating room and on the utility of the video files themselves.

\section{Results}

Table 1 lists the eight different surgical procedures which have been filmed (Figure 2) along with procedures we plan to film. For the laparoscopic procedures, GoPro recording (for port placement and positioning) was combined with the internal, laparoscopically recorded component. Overall, the resolution and framerate of the video files yielded high quality recordings of each operation.

Teaching of surgical techniques provided by staff urologists during the procedures was captured using the GoPro. When open feedback was solicited, surgeons did not report head constriction or difficulties during movement with the camera head mount.

Key steps in each procedure were highlighted with timestamps in the description of each video once uploaded to Microsoft SharePoint.

The survey was sent to 12 residents and 12 staff using Google Forms. 100\% response rate was achieved. $91 \%$ of the residents agreed that they planned on using the surgical video database. Amongst the residents, the most common resource reported for preparing for surgery was Hinman's Atlas of Urologic Surgery. The second most common resource was YouTube. All residents agreed that the database will improve their learning in the operating room based on videos accrued so far. Of the residents who had already used the database, all of them felt more confident and well-prepared for cases after using the surgical recordings when compared to their typical resources used for preparation (YouTube, Hinman's). All attendings surveyed agreed that the database will help residents prepare for procedures.

\section{Discussion}

The exposure of Canadian urology residents to open surgical procedures is decreasing both due to increased use of minimally invasive techniques, ${ }^{2}$ and overall decreases in operating room exposure. ${ }^{9}$ New methods of teaching residents have been developed, one of which includes the 
recording and reviewing of surgical procedures. Unfortunately, many of these video libraries are solely laparoscopic/robotic based. ${ }^{1}$ These new teaching methods have been formalized for some programs ${ }^{7}$ but for others it is a practice that the residents are pursuing on their own accord (e.g. YouTube). ${ }^{10}$

One of the first papers on this topic described using a GoPro for orthopedic cases and found the camera performed well, but the authors did not recommend it for high quality microsurgery videos. ${ }^{11}$ Since then, better GoPro devices have been released, and more recent studies in other specialties have reported widespread satisfaction for open surgical procedures recording. ${ }^{3-8}$

The current experience with the GoPro has been similarly encouraging. Resident feedback has been overwhelmingly positive. Being able to listen to staff narrate teaching points and steps during the recordings gives useful context to the videos. Adding multiple recordings of the same surgery, with variations in technique that come with changes in patient anatomy and or disease, has added deeper understanding. GoPro footage of port placement during laparoscopic procedures have been invaluable. Port placement is well described in many urological anatomy textbooks, however differences in body habitus and mass size can introduce challenges not discussed in these reference materials. Reviewing these port placement videos, and being able to correlate them with the internal recordings from the laparoscopic tower has improved the residents' comfort-level during surgical procedures based on informal feedback solicited from residents who have used the database thus far. We hope our database will replace YouTube as a more accurate and comprehensive resource for residents to prepare for open procedures.

Microsoft SharePoint provides a simple, secure, and collaborative interface to store the collection of surgical videos. Each resident can leave comments and pose questions in the comments section for other residents or staff to see and respond to.

The GoPro setup and recording does have room for improvement. The videos have the rolling horizontal line artifact, but it was not considered bothersome enough to modify the GoPro device according to the residents who have used the database. Aligning the GoPro took some time before the case, and for recordings that were improperly aligned, further instruction was provided to residents to ensure proper camera positioning prior to each procedure with the help of the GoPro phone application. There was a small learning curve to using the editing software that may discourage the technological neophyte from this endeavor. Finally, standard OR lighting was found to be inadequate for any deep surgery through a small incision. This would likely be ameliorated by a recording headset that includes a headlamp, as reported by others. ${ }^{3}$

Our long-term goal is to create a comprehensive video library of open surgical procedures performed at our center. This will enable residents to further prepare for each surgery while understanding the nuances in surgical procedure associated with each individual attending. To our knowledge, we are the first Canadian residency program that has begun methodically collecting videos of open surgical procedures. Our experience has been positive, and we believe this is a worthwhile investment for any surgical residency program. A national shared repository 
may be more comprehensive and allow for the exchange of surgical techniques across urology programs. A more formal evaluation of our residents' performance and preparation in the operating room using the surgical videos will be assessed once the database has matured.

\section{Conclusions}

The field of urology has long embraced the implementation of new technology into its surgical practice. Open surgical recordings have lagged behind those done for laparoscopy or robotics in volume and breadth. The GoPro camera offers a low-cost, high-quality option to record and collect open surgical videos that can be reviewed by residents for a greater learning experience.

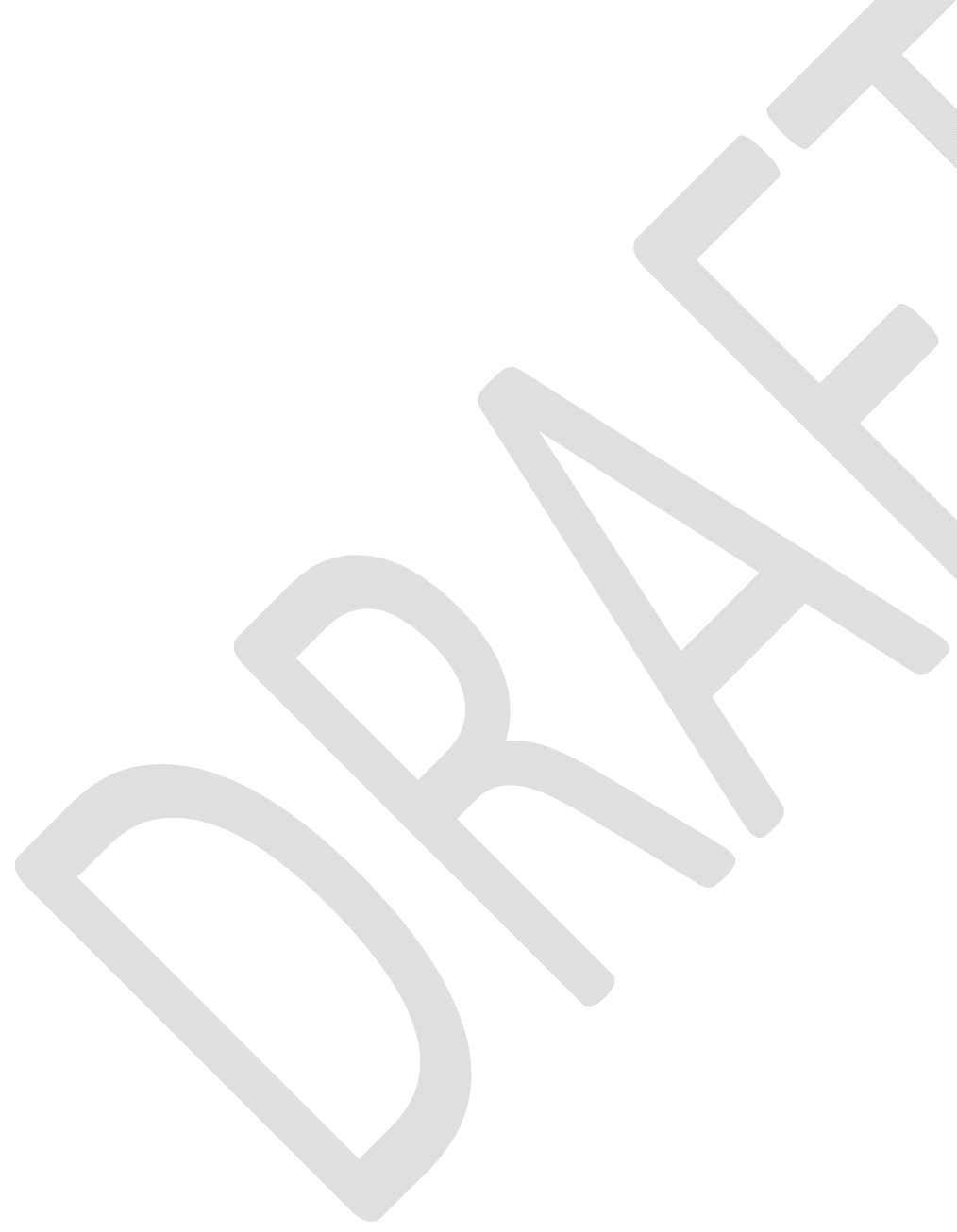




\section{References}

1. American Urological Association: Surgical Video Library. 2020. Available at: https://auau.auanet.org/content/surgical-video-library-2020.

2. Mamut AE, Afshar K, Mickelson JJ, et al: Surgical case volume in Canadian urology residency: a comparison of trends in open and minimally invasive surgical experience. $J$ Endourol 2011; 25: 1063-1067.

3. Ganry L, Sigaux N, Ettinger KS, et al: Modified GoPro Hero 6 and 7 for Intraoperative Surgical Recording-Transformation Into a Surgeon-Perspective Professional Quality Recording System. J Oral Maxillofac Surg 2019; 77: 1703.e1-1703.e6.

4. Paro JAM, Nazareli R, Gurjala A, et al: Video-based self-review: comparing Google Glass and GoPro technologies. Ann Plast Surg 2015; 74 Suppl 1: S71-4.

5. Graves SN, Shenaq DS, Langerman AJ, et al: Video Capture of Plastic Surgery Procedures Using the GoPro HERO 3+. Plast Reconstr surgery Glob open 2015; 3: e312.

6. Lin LK: Surgical video recording with a modified GoPro Hero 4 camera. Clin Ophthalmol 2016; 10: 117-119.

7. Karam MD, Thomas GW, Taylor L, et al: Value Added: the Case for Point-of-View Camera use in Orthopedic Surgical Education. Iowa Orthop J 2016; 36: 7-12.

8. Moore MD, Abelson JS, O'Mahoney P, et al: Using GoPro to Give Video-Assisted Operative Feedback for Surgery Residents: A Feasibility and Utility Assessment. J Surg Educ 2018; 75: 497-502.

9. Kairys JC, McGuire K, Crawford AG, et al: Cumulative Operative Experience Is Decreasing During General Surgery Residency: A Worrisome Trend for Surgical Trainees? J Am Coll Surg 2008; 206: 804-811. Available at: http://www.sciencedirect.com/science/article/pii/S1072751508002135.

10. Mota P, Carvalho N, Carvalho-Dias E, et al: Video-Based Surgical Learning: Improving Trainee Education and Preparation for Surgery. J Surg Educ 2018; 75: 828-835.

11. Bizzotto N, Sandri A, Lavini F, et al: Video in Operating Room: GoPro HERO3 Camera on Surgeon's Head to Film Operations-A Test. Surg Innov 2014; 21: 338-340. Available at: https://doi.org/10.1177/1553350613513514. 
Figures and Tables

Fig. 1. Photo of GoPro head mount in use. Photo credit Landan MacDonald.

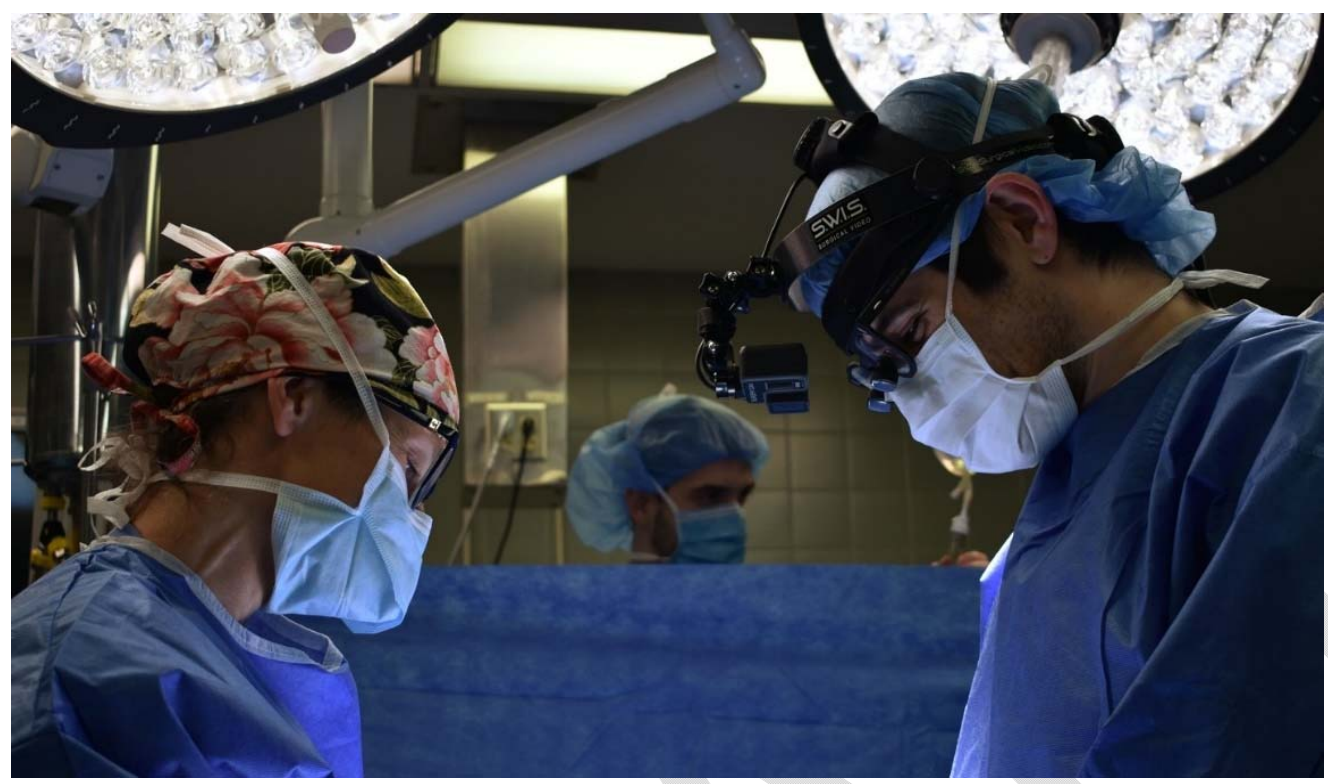

Fig. 2. Examples of procedures captured using the GoPro device: (A) retroperitoneal lymph node dissection (RPLND); (B) artificial urethral sphincter; (C) orchidopexy; and (D) penile implant.
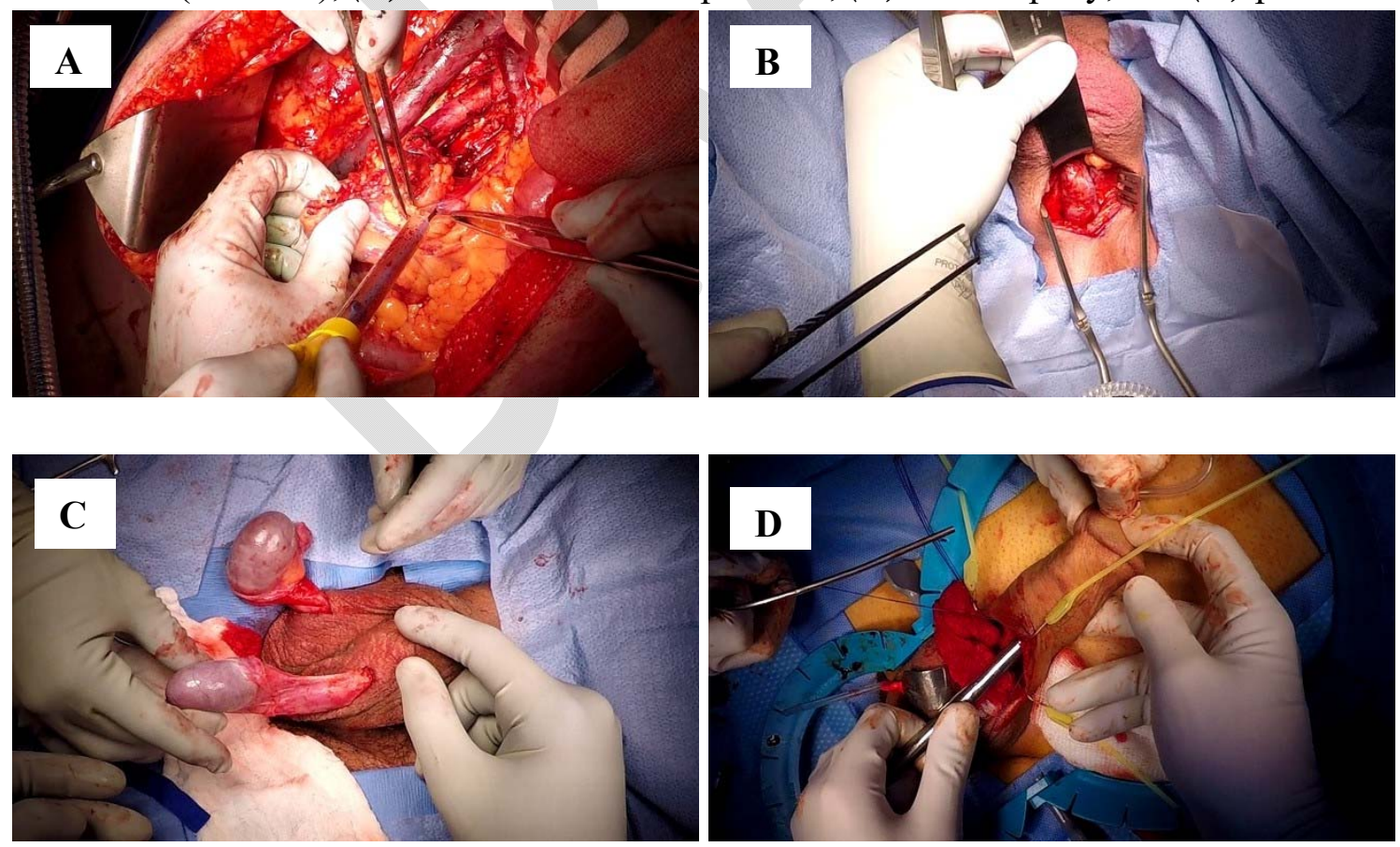


\begin{tabular}{|l|l|}
\hline Table 1. List of current and planned recordings of open surgical procedures \\
\hline Current & Planned \\
\hline Laparoscopic adrenalectomy (left and right) & Bladder augmentation \\
\hline Orchidopexy & Kidney transplant \\
\hline Retroperitoneal lymph node dissection & Vasectomy \\
\hline Partial nephrectomy (left) & Hydrocelectomy \\
\hline Artificial urinary sphincter & Varicocelectomy \\
\hline Inflatable penile prosthesis & Radical orchiectomy \\
\hline Laparoscopic nephroureterectomy (left) & Penectomy \\
\hline Laparoscopic partial nephrectomy (right) & Pyeloplasty \\
\hline & Tension-free vaginal tape (TVT) sling \\
\hline & Pubovaginal sling \\
\hline & Boari flap \\
\hline & Psoas hitch \\
\hline & Nephrectomy \\
\hline & \\
\hline
\end{tabular}

